\title{
Determinants in Human Gait Recognition
}

\author{
Tahir Amin*, Dimitrios Hatzinakos \\ Department of Electrical and Computer Engineering, University of Toronto, Toronto, Canada \\ Email: ${ }^{\prime}\{$ tamin, dimitris\}@comm.utoronto.ca
}

Received December 29, 2011; revised January 31, 2012; accepted February 20, 2012

\begin{abstract}
Human gait is a complex phenomenon involving the motion of various parts of the body simultaneously in a 3 dimensional space. Dynamics of different parts of the body translate its center of gravity from one point to another in the most efficient way. Body dynamics as well as static parameters of different body parts contribute to gait recognition. Studies have been performed to assess the discriminatory power of static and dynamic features. The current research literature, however, lacks the work on the comparative significance of dynamic features from different parts of the body. This paper sheds some light on the recognition performance of dynamic features extracted from different parts of human body in an appearance based set up.
\end{abstract}

Keywords: Biometrics; Feature Comparison; Feature Extraction; Gait Recognition

\section{Introduction}

The recognition of people by their physiological or behavioral characteristics is called biometrics. The use of biometrics in personal identification is not new and it has been used in criminology for a long time. Fingerprint databases are widely used by the law enforcement agencies from the early 19th century. Biometrics such as artistic drawings of facial features, color of eyes and footprints were routinely used in criminology even before the advent of modern technology. The invention of modern computers and digital technology have, however, transformed the way information is stored and processed. Digital technology has enabled us to store and process biometric data automatically without intervention or with our minimal input. The renewed focus on security in the past few years has brought the biometrics research into limelight. Recent developments in the biometric research have brought face, iris and fingerprint recognition from research labs to daily life. Biometric recognition systems are being installed as access control systems for granting access to offices, residential buildings and even laptop computers.

Gait is a behavioral biometric which can be perceived from a distance. It can be acquired without personal contact and cooperation. Iris and face biometrics have similar advantages but they need high resolution images and frontal view. However, it is possible to extract gait patterns from low resolution images. Human gait can vary over long durations due to many factors such as change

${ }^{*}$ Corresponding author. in body weight, injuries and disease. However studies have indicated that it still possesses sufficient discriminatory power for personal recognition [1]. Gait is a complex function of skeletal structure, muscular activity, body weight, limb lengths, bone structures etc. This complexity of gait renders it difficult to imitate and hide if not impossible.

Human gait analysis can be used as a useful tool in a variety of applications. One such promising application is medical diagnostics of diseases that affect voluntary muscle activity such as walking. For example, Parkinson's disease that affects nerve cells in part of the brain controlling muscle movements. People with Parkinson's often experience trembling, muscle rigidity, difficulty in walking, and problems with balance and coordination. Early detection of walking disorders by motion analysis can be very helpful for the treatment of such diseases. Gait can also be used to generate early warning for law enforcement agencies by detecting suspicious motion activity in airports or subway stations.

The earliest work on human motion perception was performed by Johansson [1]. He used Moving Light Display (MLD) to study human motion perception. In his experiments, movements of bright spots on a display created impressions of walking, dancing and running persons to the viewers. Human gait analysis from digital video data can be broadly categorized into two classes; model based and appearance based. Model based approaches assume a priori geometric shape model of human body while no such assumption is taken in case of appearance based approaches. Initialization of human body model is performed at the start of tracking process in model 
based approaches. The majority of techniques use manual initialization of the model in the first frame with fixed lengths of body segments. There are no such initialization issues in appearance based methods. However, the appearance based method being holistic in nature can suffer from lack of correspondence between extracted gait signatures to the actual physical quantities. Figure 1 shows the block diagram of a generic gait identification system.

The most critical step in gait recognition system is the extraction of gait features from video data. Human gait is cyclic in nature and this characteristic exhibits itself in cyclic appearance changes in the images when taken from a side view. Although gait is a dynamic process, studies have shown that static body parameters such as length and widths of limbs are also important in gait recognition. In appearance based methods, dynamics of lower half of the body are generally considered more important. Studies have been performed on the relative importance of static and dynamic features in gait recognition. But there is a lack of work in relative analysis of dynamic features from different parts of the body especially in an appearance based set up. Dynamics of different parts of the human body play a role in characterizing the human gait pattern. This paper analyzes the discriminatory power of features extracted from different parts of the body by applying area masks.

The rest of the paper is organized as follows. Section 2 gives a brief overview of the previous works in gait feature comparative analysis. In Section 3, we discuss preprocessing of silhouettes and provide details of dynamic feature extraction. Experimental results and discussion are given in Section 4. Section 5 draws conclusions with some directions to the future work. References are listed in Section 6.

\section{Previous Works}

We will briefly review the previous works on determination of characteristics that contribute to gait recognition and their relative importance. As mentioned earlier in the

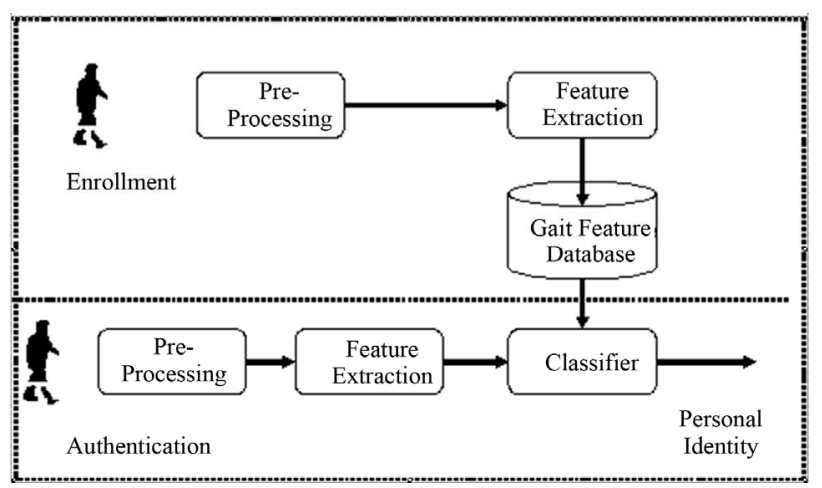

Figure 1. Block diagram of the gait recognition system. preceding section, the current research literature lacks the comparative analysis of dynamic features extracted from different parts of the body. The comparison between static and dynamic features has, however, been studied in some of the works which will be summarized below.

Human locomotion has been widely studied by medical and physiological research community. Their main purpose is to determine the gait variations and distinguish between normal and pathological gait and rehabilitation of patients. Saunders et al. have defined human walking as the translation of the center of mass of the body from one point to another in a way that requires the least energy [2]. They also identified six gait determinants or variables that affect the energy expenditure. The six gait determinants are pelvic rotation, pelvic tilt, knee flexion at mid-stance, foot and ankle motion, knee motion and lateral pelvic displacement. The focus of this work as well as other similar ones was to study the movement of different muscles and limbs during the gait process. These types of studies are useful for detecting abnormalities in human walking and may also serve as a general guideline for recognition systems. The perception of human gait as well as its recognition involves much more than just the six determinants given in [2].

Wang et al. used both static and dynamic body biometrics for human recognition [3]. The static body features were derived from using the Procrustes shape analysis to obtain a compact appearance representation. The dynamic descriptors were estimated by recovering joint angle trajectories of the lower limbs using Condensation algorithm. The algorithm was evaluated by using a database consisting of 80 sequences from 20 subjects and four sequences per subject. They reported recognition of $83.75 \%$ at rank 1 by using only static features and a success rate of $87.5 \%$ when dynamic features were used. The combined features resulted in an increased recognition rate of $97.5 \%$ at rank 1 . Wang et al. also performed comparative study between the dynamic and static features in their work reported in $[3,4]$. Their work showed that the dynamic information extracted from the video sequences is somewhat better for human identification than the static information. In [5], BenAbdelkader, Cutler and Davis, proposed a parametric technique for personal identification. Their gait signatures are based on the height and stride parameters extracted from low resolution video sequences. The experimental evaluations were performed on a database containing 45 subjects. A recognition rate of $49 \%$ was achieved by using both stride and height parameters and only $21 \%$ by using just the stride parameter. In [6], Bobick and Johnson used 4 static body parameters and averaged them to get mean walk vector for each gait sequence. The recognition results of this method are available in the form of CMC plots at the Georgia Tech human identification website [7]. Although 
the method is able to achieve a very compact representation of human gait (4-dimensional), its performance is low for lower rank recognition.

Veeraraghavan et al. [8] conducted a detailed comparison between shape and kinematic features for human recognition. Their experiments indicated that shape of the body carries more information than the kinematics for recognition of humans from video sequences. However, using kinematics in conjunction with shape features considerably improved the performance of the system. Similarly, gait analysis work carried out by R. Green and L. Guan also showed that anthropometric (static) features extracted by them were more discriminatory for human identification than the dynamic features in the shape of joint angle trajectories [9]. On the other hand, the experiments conducted by Johansson establish the importance of dynamic features for identification [1]. Contradicting results have been reported about the importance of dynamic and static features while dynamic feature comparison has not been performed explicitly. This work is poised to shed some light on the dynamic feature performance extracted from different parts of the binary silhouettes.

\section{Extraction of Body Dynamics}

In appearance based gait recognition systems, the first step usually involves segmentation of human subject from the background. This segmentation process is usually performed by background subtraction. The resulted silhouettes are noisy because of segmentation errors. We observe that silhouettes in the database contain outliers which should be removed to make the gait feature extraction more robust. We use median filtering with a mask of $5 \times 5$ to filter the silhouettes to get rid of outliers. The output from the median filter is binarized by simple thresholding to obtain smoothed silhouettes.

Human walking process is cyclic in nature. Gait cycle is the time between two identical events during human walking. It is usually measured from heel strike to heel strike of one leg. A complete gait cycle is shown in Figure 2.

The movement of arms and legs is the most prominent motion during gait cycle. Assuming that image plane is perpendicular to the direction of motion, the gap between

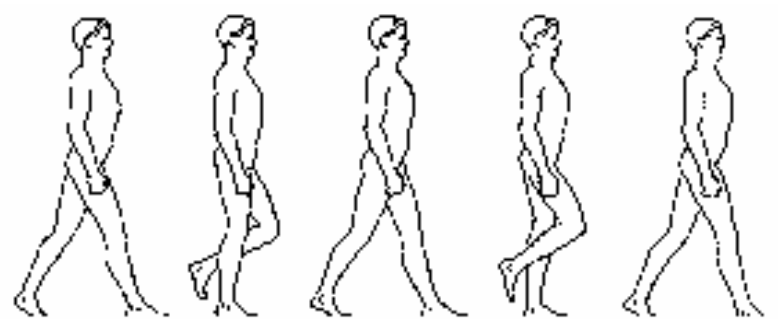

Figure 2. Human gait cycle. two legs in 2D silhouettes changes during gait cycle. Similarly the gap between two arms and the rest of the body also changes in a cyclic fashion. This dynamic information can be captured by applying area masks at different parts of the binary silhouettes similar to the approach adopted by Foster et al. [10]. The number of pixels of the binary silhouettes under these masks is calculated. The process is repeated for each binary silhouette in the gait sequence and we obtain six area signals of length $N$, the number of frames in the gait sequence. The width of each area mask is 15 pixels. Figure 3 shows the location of six area masks for an example silhouette from the database.

The following equations summarize the extraction of six area signals from the masks shown in Figure 3.

$$
\begin{gathered}
b[i, j]=\left\{\begin{array}{cc}
1 & \text { if pixel }[i, j] \text { belongs to foreground } \\
0 & \text { if pixel }[i, j] \text { belongs to background }
\end{array}\right. \\
m_{p}[i, j]=\left\{\begin{array}{cc}
1 & \text { for } L_{p} \leq i<L_{p} \\
0 & \text { otherwise }
\end{array}\right. \\
a_{p}(n)=\sum_{i, j} b_{n}[i, j] m_{p}[i, j]
\end{gathered}
$$

where $b[i, j]$ is the binary silhouette, $m_{p}[i, j]$ is the area mask and $a_{p}(n)$ is the area under mask $p$ for frame $n$ of the silhouette sequence. $L_{p}$ is the starting row for mask $m_{p}$ and $p=[1, \cdots, 6]$ is the mask index. These area signals are shown in Figures $\mathbf{4}$ and $\mathbf{5}$ for two typical silhouette sequences from the database.

The area signals extracted by applying the area masks are noisy due to the imperfections in the silhouette extraction process. It is observed that a high frequency riding wave is present in all of the area signals. We apply a newly proposed Empirical Mode Decomposition (EMD) algorithm to remove these riding waves to get cleaner area signals. EMD algorithm is described in the following subsection.

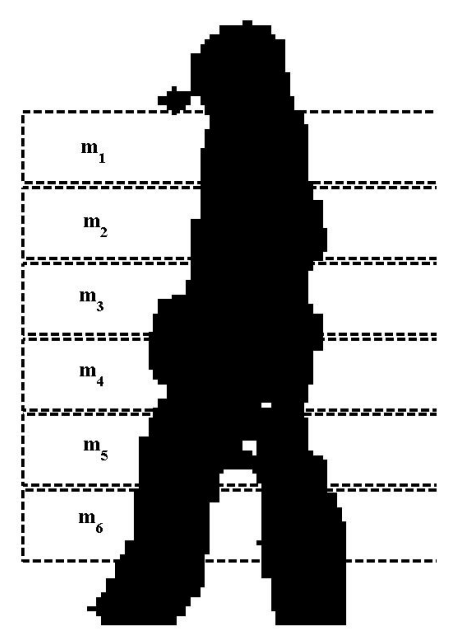

Figure 3. Area masks. 


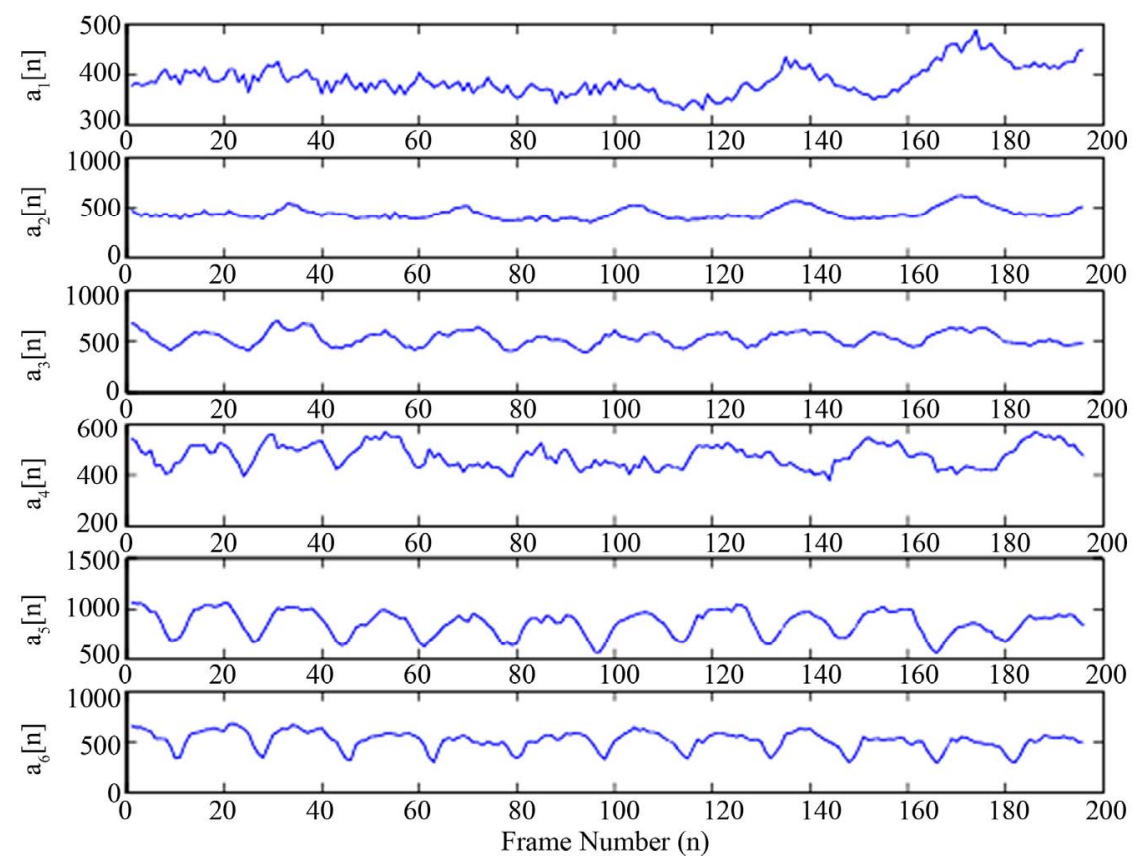

Figure 4. Area signals for a silhouette sequence.
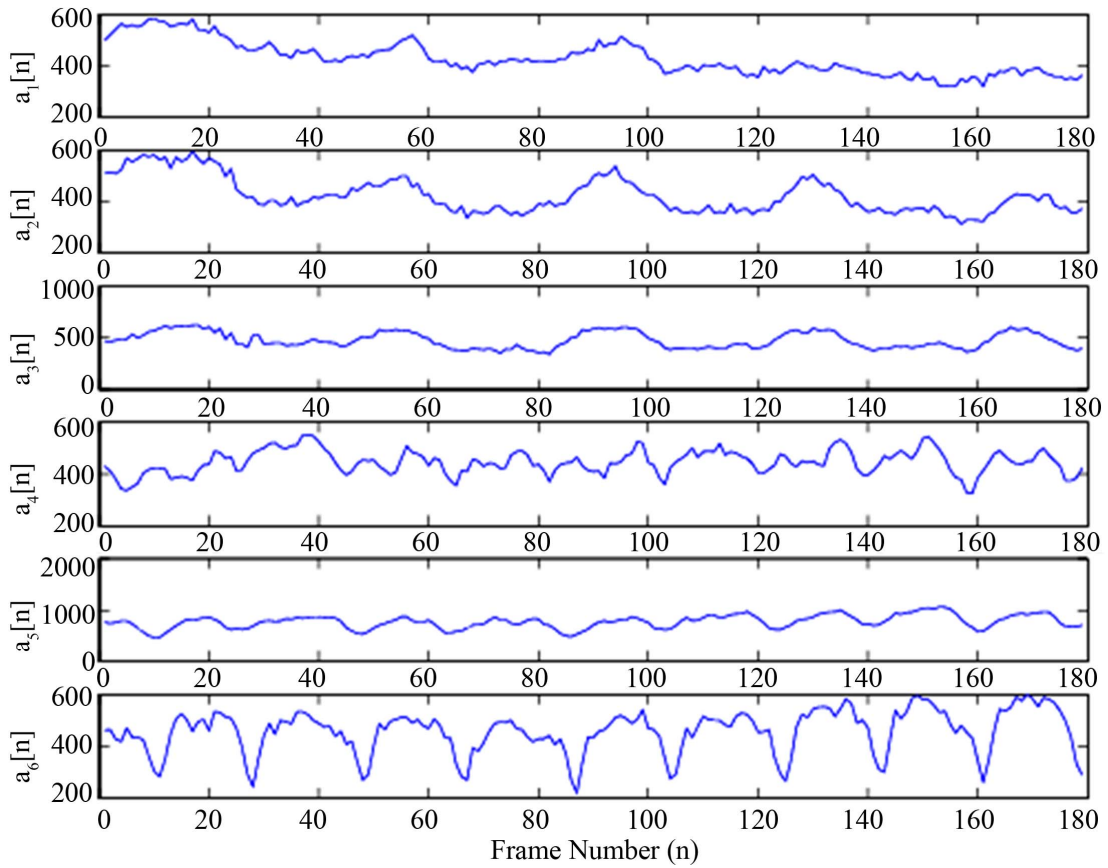

Figure 5. Area signals for another silhouette sequence.

\subsection{Empirical Mode Decomposition (EMD)}

The traditional data analysis methods such as Fourier transform have an inherent restriction to their application. They are suitable when the system is linear and the data is stationary. In most of the practical application scenarios these two conditions are rarely satisfied. But these traditional methods are still widely used because of their simplicity and well formed theoretical basis. In some cases, non linear and non stationary data can be transformed to linear and stationarity data before processing it with Fourier based methods. But in other cases new methods are needed which can analyze non linear and non stationary data. There has been some progress in the analysis of non stationary data in recent years. Wavelet analysis and Wagner-Ville distribution are the examples of data analysis tools for non stationary data. Huang et al. 
proposed EMD to decompose non linear non stationary data into oscillatory modes called Intrinsic Mode Functions (IMF) [12]. The method separates IMFs from signals modulated in both amplitude and frequency. IMF is a function that satisfies two conditions:

- The number of extrema and the number of zero crossings are either equal or at most differ by one.

- The mean value of the envelope traced by the local maxima and the envelope defined by the local minima is zero.

IMFs are extracted by the sifting process which is applied iteratively until a predefined condition is satisfied or the residue becomes a monotonic function. The signal $\mathrm{x}(\mathrm{t})$ can be then represented in the following form:

$$
a(t)=\sum_{i=1}^{k} e_{i}+r_{k}
$$

where $e_{i}$ denotes the $i$ th extracted empirical mode and $r_{k}$ is the residue which is either a constant or mean trend. The sifting procedure to obtain the IMF is summarized in 6 steps as given in Algorithm 1.

\section{Algorithm 1: EMD Algorithm}

1) Extract all local extrema.

2) Determine the upper envelope by connecting all the local maxima by cubic spline interpolation.

3) Determine the lower envelope by connecting all the local minima by cubic spline interpolation.

4) Calculate the mean envelope $m_{1}$ from upper and lower envelopes.

5) Calculate the first component $h_{1}$ as follows:

$$
h_{1}=x(t)-m_{1}
$$

6) Check if $h_{1}$ satisfies the IMF definition.

a) If yes:

$$
e_{1}=h_{1}
$$

Calculate residue as follows:

$$
r_{1}=x(t)-h_{1}
$$

Go to step 1 and repeat the sifting process to extract more IMFs treating $r_{1}$ as the input data.

b) if no: Calculate $h_{11}$

$$
h_{11}=h_{1}-m_{11}
$$

Where $m_{11}$ is the mean envelope of $h_{1}$. Repeat the sifting $k$ times until the stoppage criterion is met to get $h_{1} k$.

$$
\begin{gathered}
h_{1 k}=h_{1(k-1)}-m_{1 k} \\
e_{1}=h_{1 k}
\end{gathered}
$$

In order to illustrate the noise removal by EMD, we chose two area signals from Figures 4 and 5 and decomposed them using the EMD algorithm. The input signals are plotted in Figure 6 and their IMFs in Figures 7 and $\mathbf{8}$.

The high frequency noise appears as the first IMF, $\mathrm{IMF}_{1}$ as shown in Figures 7 and $\mathbf{8}$. The area signals are reconstructed by ignoring $\mathrm{IMF}_{1}$ as given in the following equations.

$$
\begin{aligned}
& a_{1}^{\prime}(n)=\sum_{i=2}^{5} e_{i}[n]+r_{k}[n] \\
& a_{2}^{\prime}(n)=\sum_{i=2}^{5} e_{i}[n]+r_{k}[n]
\end{aligned}
$$

The reconstructed signals are shown in Figure 9.

\subsection{Correlation Analysis}

After the noise removal from area signals by EMD algorithm, we compute autocorrelation of all six reconstructed area functions as follows:

$$
R_{a_{p}^{\prime}}[l]=\sum_{n} a_{p}^{\prime}[n] a_{p}^{\prime}[n+l]
$$

where $R_{a_{p}}$ represents the autocorrelation function of the reconstructed area signal $a_{p}^{\prime}$ and $l$ is the time lag. $R_{a_{p}}$ is only calculated for positive lags i.e. $l=[1 . . N]$. The dynamic gait features are then derived by taking the Discrete Cosine Transform (DCT) of the autocorrelation functions. The DCT of a discrete function $R_{a_{p}^{\prime}}$ is defined as:

$$
T_{p}(k)=c(k) \sum_{l=0}^{N-1} R_{a_{p}^{\prime}}(l) \cos \left(\frac{\pi(2 l+1) k}{2 N}\right)
$$

where $T_{p}(k)$ is the DCT transform of the original signal $R_{a_{p}}(l)$ of length $\mathrm{N}$. The coefficient $c(k)$ is given by:

$$
c(0)=\sqrt{\frac{1}{N}}, \quad c(k)=\sqrt{\frac{2}{N}}
$$

for $1 \leq k \leq M-1$.
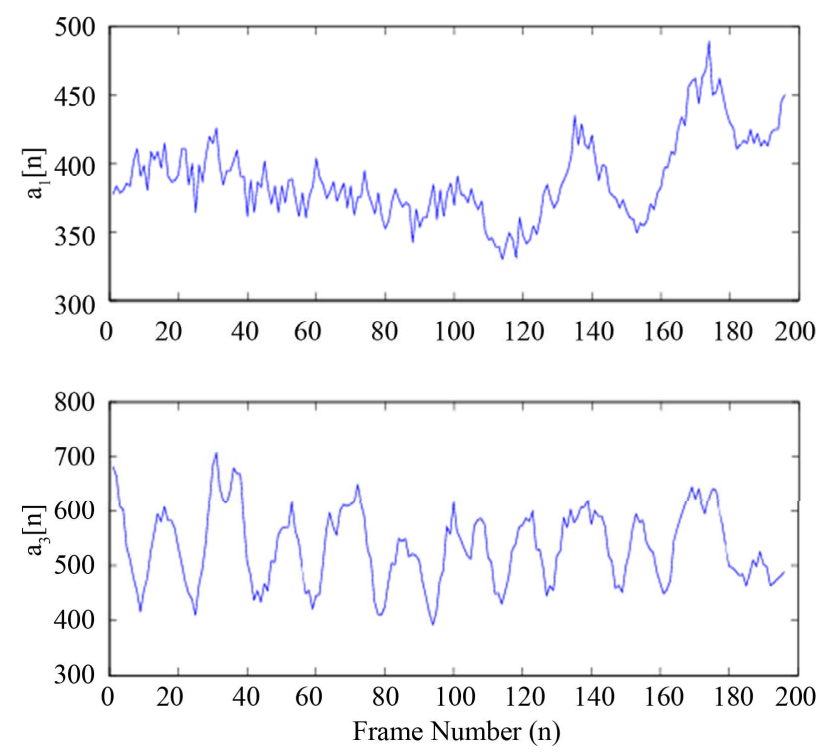

Figure 6. Noisy area Signals. 


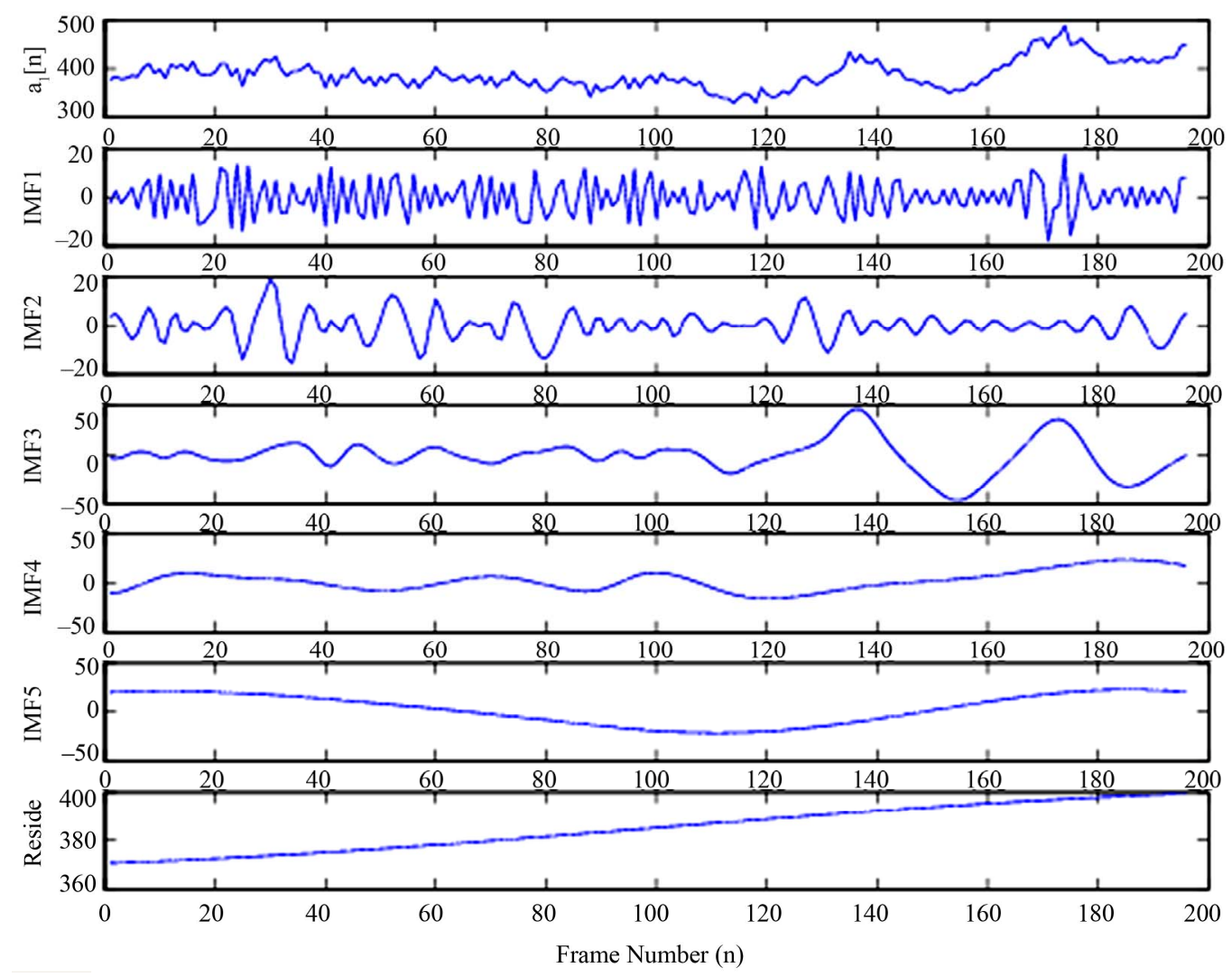

Figure 7. Input signal $a_{1}$ and its IMFs.
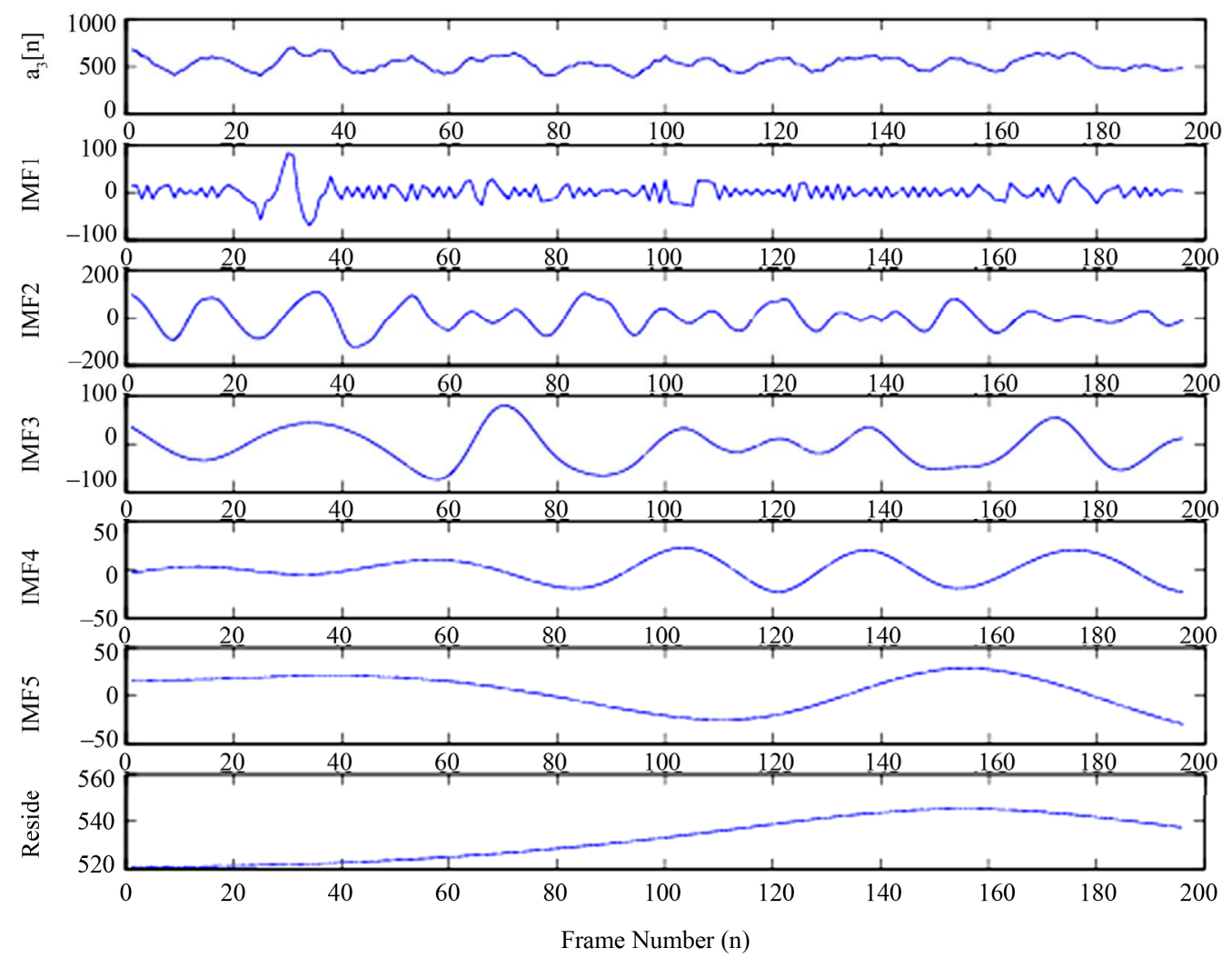

Figure 8. Input signal $a_{3}$ and its IMFs. 

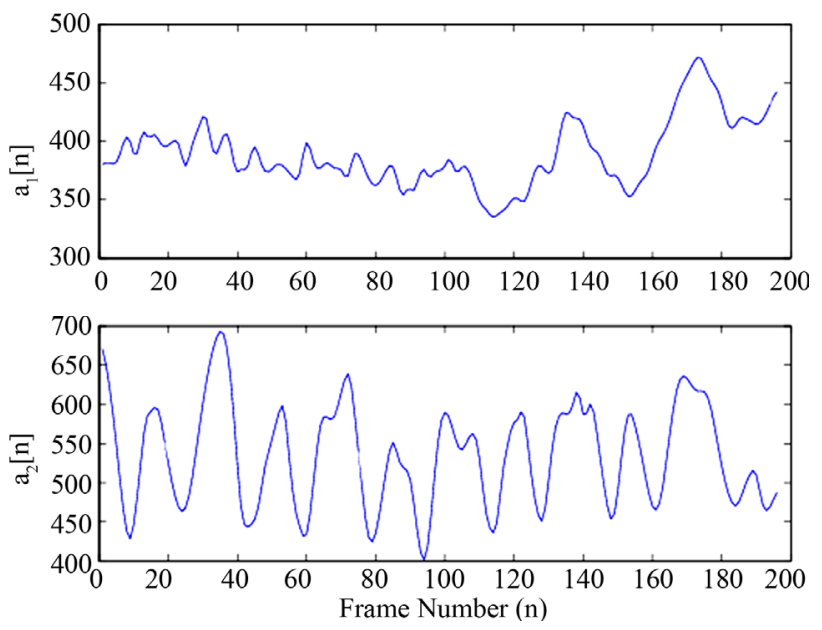

Figure 9. Area signals after noise removal.

\section{Experimental Results}

\subsection{Database Description}

We use the May 2001 version of Gait Challenge (GC) database from University of Southern Florida (USF) [12]. This database consists of 452 sequences from 71 subjects recorded under the following 5 covariates.

- shoe type, A or B

- surface type, Grass (G) or Concrete (C)

- carrying conditions, with Briefcase (BF) or without (NB)

- viewpoints, Left (L) or Right (R)

- time instants, $t_{1}$ or $t_{2}$

In order to assess the performance of gait recognition algorithms, the GC database also contains Human ID Challenge Experiments. There are total 11 experiments. We use experiment A (G, A, L, NB, $\left.t_{1}\right)$ to analyze the recognition potential of dynamic features extracted from different parts of the silhouettes. Experiment A is chosen due to the following reasons:

- Both Gallery (G, A, R, NB, $\left.\mathrm{t}_{1}\right)$ and A set contain all the 71 subjects

- They are recorded under similar conditions except a different viewpoint. This eliminates the effect of other covariates which can skew the results.

\subsection{Gait Features}

Each silhouettes sequence is processed frame by frame for the extraction of dynamic gait features. The silhouette frames are processed by median filtering to reduce outliers. Next, we estimate the gait period from the autocorrelation function of the silhouette area signal. Speed normalization is achieved by ensuring the same number of frames in each gait cycle for all silhouette sequences. The six area signals are then extracted as explained in the preceding section and EMD algorithm is applied to reduce the noise. DCT coefficients of autocorrelation functions of each of the six reconstructed area signals are computed. The first 35 DCT coefficients form our dynamic gait feature.

\subsection{Feature Vector Normalization}

We normalize the gait features by using Equation 16 to put equal emphasis on each component of the feature vector. Each of the components of 35-dimensional feature vector takes on different value for each silhouette sequence. These different values of each component form a sequence of numbers. We consider that these sequences are being generated by a Gaussian distribution. Mean $\mu_{v}$ and standard deviation $\sigma_{v}$ of each such sequence representing a component of the gait signature $v$ are calculated and then the sequence is normalized as follows:

$$
v_{i}=\frac{v_{i}-\mu_{v}}{\sigma_{v}}
$$

This normalization process maps most of the values of the feature components $v$ in the range $[-1,1]$. The advantage of this normalization is that a few abnormal values occurring in the sequence will not bias the importance of other values.

\subsection{Feature Matching}

Features are matched by nearest neighborhood using the simple City block distance. The City block distance between the two gait feature vectors is defined as:

$$
d(\mathbf{p}, \mathbf{g})=\sum_{i=1}^{P}\left|p_{i}-g_{i}\right|
$$

where $\mathbf{p}$ and $\mathbf{g}$ are the feature vectors of probe and gallery sequences respectively and $p$ is the dimension of the signature vector.

\subsection{Comparison of Dynamic Features}

Cumulative Match Score (CMS) is used to evaluate the performance of different dynamic gait features. Each probe sequence feature is compared with the features of 71 sequences in the gallery. The gallery set consists of one sequence for each subject. The gallery sequence set is sorted according to the similarity to the probe sequences. The rank1 identification result is the total number of correct matches appearing on the top while Rank 5 value represents the correct identification obtained among the top 5 matches.

Table 1 presents the performance evaluation of features extracted from the six area signals at Rank 1 and Rank 5. At Rank 1, the best performance of $97.18 \%$ is achieved from the features extracted from $a_{6}$. This area signal represents the dynamics of lower leg during the 
Table 1. Comparison of features at rank 1 and rank 5.

\begin{tabular}{ccc}
\hline Area Signal & Rank 1 & Rank 5 \\
\hline & $\mathbf{( \% )}$ & $\mathbf{( \% )}$ \\
$a_{1}$ & 53.52 & 94.37 \\
$a_{2}$ & 53.52 & 92.96 \\
$a_{3}$ & 78.87 & 100 \\
$a_{4}$ & 73.24 & 98.59 \\
$a_{5}$ & 73.24 & 98.59 \\
$a_{6}$ & 97.18 & 100 \\
\hline
\end{tabular}

gait motion. The second most significant results of $78.87 \%$ are achieved from $a_{3}$ which represents the lower arm dynamics. We achieve a recognition performance of $73.24 \%$ for both $a_{4}$ and $a_{5}$ features. Similarly, rank 1 recognition performance of $a_{1}$ and $a_{2}$ features is also the same at $53.52 \%$. Rank 1 results indicate that thigh movement and knee movement is of equal importance in gait recognition. However, the performance of thigh and knee features is slightly lower than the features extracted from the lower arm dynamics.

Similar result pattern is obtained at rank 5. $a_{6}$ and $a_{3}$ features provide the best recognition performance of $100 \%$. The recognition rate of $a_{4}$ and $a_{5}$ is slightly lower at $98.59 \%$. The lowest performance of $92.96 \%$ is obtained from $a_{2}$ features. The recognition performance of $a_{1}$ features is slightly higher than $a_{2}$ feature set at $94.37 \%$.

Figure 10 shows the CMC plot for DCT features of six area signals. The recognition performance of the dynamic features extracted from $a_{3}$ and $a_{6}$ is superior to the other feature sets. These results partially support the traditional notion of significance of leg dynamics in gait recognition. It is also observed that the dynamics of the lower arm is very important in determining the gait pattern of the human subjects in an appearance based setup.

\section{Conclusion}

Human locomotion is a complex phenomenon involving the coordination of different limbs as the body translates from one point to another. The static configuration of the body such as the widths and lengths of different limbs have been shown of great importance in determining the gait pattern of the individuals. The contribution of dynamics of different parts of human body has not been studied explicitly in an appearance based recognition set up. In this paper, we have analyzed the recognition performance of dynamic features from different parts of the body. It is shown by experimental evaluation that dynamics of lower leg and lower arm are of utmost importance for building an efficient gait recognition system. The motion of lower half of the body has always been considered more important in the determination of gait pat-

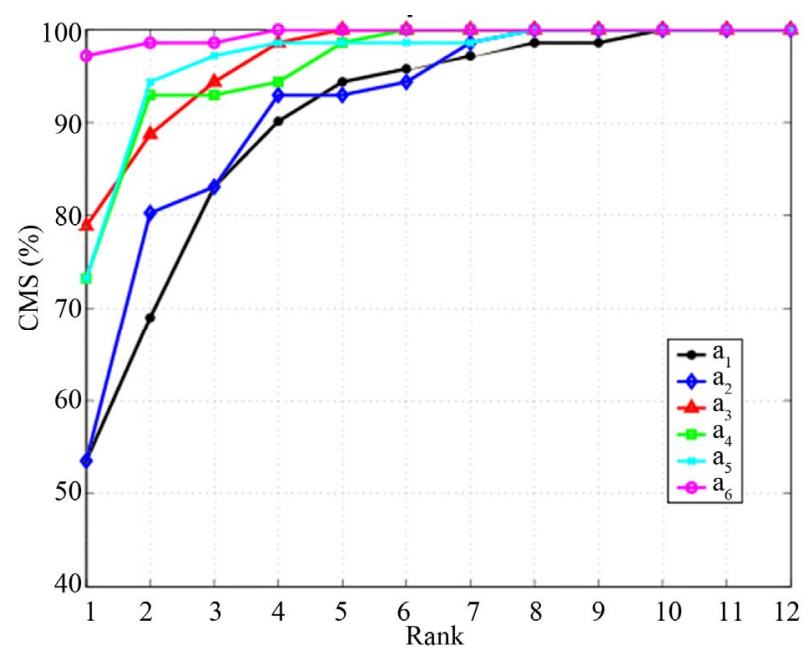

Figure 10. CMC for six area features.

tern. However, we have found that lower arm movement also plays an important role in gait recognition. The results from the present work can be used for building a better feature selection process for a more robust recognition system. Lower leg is usually very noisy in the extracted silhouettes because of shadows and walking surface issues. A set of better discriminatory features may be extracted from lower arm motion avoiding noisy data from lower leg.

\section{REFERENCES}

[1] G. Johansson, "Visual Perception of Biological Motion and a Model for Its Analysis," Attention, Perception, \& Psychophysics, Vol. 14, No. 2, 1973, pp. 210-211. doi:10.3758/BF03212378

[2] J. B. dec. M. Saunders, V. T. Inman and H. D. Eberhart, "The Major Determinants in Normal and Pathological Gait," The Journal of Bone and Joint Surgery, Vol. 35-A, No. 3, 1953, pp. 543-558.

[3] L. Wang, H. Z. Ning, T. N. Tan and W. M. Hu, "Fusion of Static and Dynamic Body Biometrics for Gait Recognition," IEEE Transactions on Circuits and Systems for Video Technology, Vol. 14, No. 2, 2004, pp. 149-158. doi:10.1109/TCSVT.2003.821972

[4] L. Wang, H. Z. Ning, T. N. Tan and W. M. Hu, "Fusion of Static and Dynamic Body Biometrics for Gait Recognition," Proceedings of the 9th IEEE International Conference on Computer Vision (ICCV 03), Nice, 13-16 October 2003, pp. 1449-1454. doi:10.1109/ICCV.2003.1238660

[5] C. BenAbdelkader, R. Cutler and L. Davis, "Person Identification Using Automatic Height and Stride Estimation," Proceedings of the IEEE 16th International Conference on Pattern Recognition, Quebec, 11-15 August 2002, pp. 377-380. doi:10.1109/ICPR.2002.1047474

[6] A. F. Bobick and A. Y. Johnson, "Gait Recognition Using Static, Activity-Specific Parameters," Proceedings of the 
2001 IEEE Computer Society Conference on Computer Vision and Pattern Recognition, Kauai, 8-14 December 2001, pp. 423-430. doi:10.1109/CVPR.2001.990506

[7] "Georgia Tech GVU Center/College of ComputingHuman Identification at a Distance," 2011. http://www.cc.gatech.edu/cpl/projects/hid

[8] A. Veeraraghavan, A. K. Roy-Chowdhury and R. Chellappa, "Matching Shape Sequences in Video with Applications in Human Movement Analysis," IEEE Transactions on Pattern Analysis and Machine Intelligence, Vol. 27, No. 12, 2005, pp. 1896-1909. doi:10.1109/TPAMI.2005.246

[9] R. D. Green and L. Guan, "Quantifying and Recognizing Human Movement Patterns from Monocular Video Images-Part II: Applications to Biometrics," IEEE Transactions on Circuits Systems for Video Technology, Vol. 14, No. 2, 2004, pp. 191-198.

\section{doi:10.1109/TCSVT.2003.821977}

[10] J. P. Foster, M. S. Nixon and A. Prügel-Bennett , "Automatic Gait Recognition Using Area-Based Metric," Pattern Recognition Letters, Vol. 24, No. 14, 2003, pp. 2489 2497. doi:10.1016/S0167-8655(03)00094-1

[11] N. E. Huang, Z. Shen, S. R. Long, M. C. Wu, H. H. Shih, Q. Zheng, N. C. Yen, C. C. Tung and H. H. Liu, "The Empirical Mode Decomposition and the Hilbert Spectrumfor Nonlinear and Non-Stationary Time Series Analysis," Proceedings of the Royal Society of London A, Vol. 454, No. 1971, 1998, pp. 903-995.

[12] S. Sarkar, P. J. Phillips, Z. Y. Liu, I. R. Vega, P. Grother and K. W. Bowyer, "The HumanID Gait Challenge Problem: Data Sets, Performance, and Analysis," IEEE Transactions on Pattern Analysis and Machine Intelligence, Vol. 27, No. 2, 2005, pp. 162-177. doi:10.1109/TPAMI.2005.39 